\title{
International Unit per Milliliter per Microgram per Day
}

National Cancer Institute

\section{Source}

National Cancer Institute. International Unit per Milliliter per Microgram per Day. NCI

Thesaurus. Code C119372.

A unit of concentration (biologic activity) equal to one international unit per milliliter, divided by micrograms per day. 